\title{
Self-powered autonomous wireless sensor node using vibration energy harvesting
}

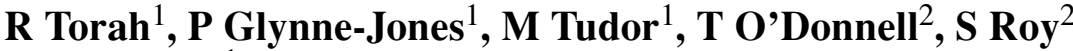 \\ and $S$ Beeby ${ }^{1}$ \\ ${ }^{1}$ School of Electronics and Computer Science, University of Southampton, UK \\ ${ }^{2}$ Tyndall National Institute, Prospect Row, Cork, Ireland
}

Received 14 May 2008, in final form 2 September 2008

Published 24 October 2008

Online at stacks.iop.org/MST/19/125202

\begin{abstract}
This paper reports the development and implementation of an energy aware autonomous wireless condition monitoring sensor system (ACMS) powered by ambient vibrations. An electromagnetic (EM) generator has been designed to harvest sufficient energy to power a radio-frequency $(\mathrm{RF})$ linked accelerometer-based sensor system. The ACMS is energy aware and will adjust the measurement/transmit duty cycle according to the available energy; this is typically every $3 \mathrm{~s}$ at $0.6 \mathrm{~m} \mathrm{~s}_{\mathrm{rms}}^{-2}$ acceleration and can be as low as $0.2 \mathrm{~m} \mathrm{~s}_{\mathrm{rms}}^{-2}$ with a duty cycle around $12 \mathrm{~min}$. The EM generator has a volume of only $150 \mathrm{~mm}^{3}$ producing an average power of $58 \mu \mathrm{W}$ at $0.6 \mathrm{~m} \mathrm{~s}_{\mathrm{rms}}^{-2}$ acceleration at a frequency of $52 \mathrm{~Hz}$. In addition, a voltage multiplier circuit is shown to increase the electrical damping compared to a purely resistive load; this allows for an average power of $120 \mu \mathrm{W}$ to be generated at $1.7 \mathrm{~m} \mathrm{~s}_{\mathrm{rms}}^{-2}$ acceleration. The ACMS has been successfully demonstrated on an industrial air compressor and an office air conditioning unit, continuously monitoring vibration levels and thereby simulating a typical condition monitoring application.
\end{abstract}

Keywords: energy harvesting, electromagnetic generator, wireless sensor, self-powered systems

\section{Introduction}

Wireless systems offer greater flexibility, increased reliability and reduced costs compared with a wired infrastructure. In addition, transmitting the data wirelessly presents the possibility of embedding the sensors in previously inaccessible locations. However, the majority of sensor nodes are reliant on battery power. These batteries will require periodical replacement and are therefore not consistent with the concept of a permanent fully embedded system.

The development of ultra low power electronics, MEMS and RF communications has encouraged the development of a number of alternative power sources. Many of these solutions involve the use of chemical energy such as fuel cells [1] and microturbine generators [2], but these require periodic refuelling. Other solutions extract energy from the environment. For example, light, thermal and/or kinetic energy can be converted into electrical energy that can augment an existing battery supply or replace it entirely given appropriate conditions.
This paper describes the development of a complete autonomous wireless condition monitoring sensor system (ACMS) powered by vibration energy harvesting. A kinetic energy generator has been developed which converts mechanical energy, in the form of vibrations, into electrical energy using an electromagnetic transduction mechanism. Previous work in this area has demonstrated generators operating with a wide range of accelerations and at frequencies ranging from a few $\mathrm{Hz}$ up to $\sim 10 \mathrm{kHz}$ [3-5]. Practical acceleration levels have been identified as varying from 0.2 to $12 \mathrm{~m} \mathrm{~s}^{-2}$ in locations such as car engine compartments and building air conditioning units [6]. The intended applications for the ACMS described in this paper are an office air conditioning unit and an industrial air compressor unit. The office air conditioning unit has resonant peaks between 40 and $50 \mathrm{~Hz}$ with acceleration levels between 0.85 and $1.7 \mathrm{~m} \mathrm{~s}^{-2}$. The vibration spectrum measurements for the air compressor are consistent with that of the air conditioning unit [7]. The generator and sensor system have therefore been designed to work initially with these applications and at low acceleration 
Table 1. Summary of mk1, mk2 and mk3 generator key components and results.

\begin{tabular}{|c|c|c|c|c|c|c|}
\hline $\begin{array}{l}\text { EM } \\
\text { generator }\end{array}$ & $\begin{array}{l}\text { Magnet } \\
\text { size }(\mathrm{mm})\end{array}$ & $\begin{array}{l}\text { Coil turns/wire } \\
\text { diameter }\end{array}$ & $\begin{array}{l}\text { Coil } \\
\text { resistance }\end{array}$ & $\begin{array}{l}\text { Open-circuit } \\
\text { voltage }\end{array}$ & $\begin{array}{l}\text { Optimum load } \\
\text { resistance }\end{array}$ & $\begin{array}{l}\text { Output power at } 0.6 \mathrm{~m} \mathrm{~s}^{-2} \\
\text { into load resistor }\end{array}$ \\
\hline $\mathrm{mk} 1$ & $1 \times 1 \times 1.5$ & $600 / 25 \mu \mathrm{m}$ & $91.6 \Omega$ & $34 \mathrm{mV}_{\mathrm{rms}}$ & $100 \Omega$ & $4.3 \mu \mathrm{W}_{\mathrm{rms}}$ \\
\hline $\mathrm{mk} 3$ & $3.7 \times 2 \times 1.5$ & $2800 / 12 \mu \mathrm{m}$ & $2323 \Omega$ & $1141 \mathrm{mV}_{\mathrm{rms}}$ & $15 \mathrm{k} \Omega$ & $58 \mu \mathrm{W}_{\mathrm{rms}}$ \\
\hline
\end{tabular}

levels, but the principles of operation can be applied to various applications. These vibration levels and frequencies are representative of many industrial applications and therefore make this an ideal demonstration of the system's capabilities. Commercially available systems [8] are typically orders of magnitude larger in size than that presented here. The challenge addressed in this paper is how to develop a generatorbased system that can extract demonstrably useful amounts of power in the available volume. Throughout this paper, all acceleration levels given are an RMS (root mean square) value unless otherwise stated.

\section{Electromagnetic generator}

The latest results presented in this paper are for the Mark 3 version of an electromagnetic generator developed during an EU funded research project 'Vibration Energy Scavenging (VIBES)'. The Mark 3 generator demonstrates a significant improvement over previous Mark 1 and Mark 2 generators, particularly in terms of voltage output. Section 3 discusses how these improvements have enabled the implementation of a complete self-powered working condition monitoring system.

The cantilever generators described here are a miniaturized form of a previous design developed at the University of Southampton [9]. The design uses four neodymium iron boron $(\mathrm{NdFeB})$ magnets configured in opposite polarities above and below a cantilever beam producing a concentrated flux gradient through a stationary copper coil as the magnets move due to environmental vibrations [10]. NdFeB rare earth magnets have the highest flux density of any commercially available magnets. The magnetic circuit is completed using zinc coated mild steel (Zintec) keepers which couple the flux between the top and bottom magnets. The magnets are mounted on a laser etched $50 \mu \mathrm{m}$ beryllium copper cantilever beam. Completing the structure are two machinable tungsten inertial masses attached to the free end of the cantilever beam using cyanoacrylate adhesive. A cantilever beam is used since it can be easily fabricated and is a space efficient structure for obtaining low resonant frequencies. The beam is $7 \mathrm{~mm}$ long from clamp to beam tip; the entire generator is $10 \mathrm{~mm}$ long with a total inertial volume of $150 \mathrm{~mm}^{3}$.

The difference between the mk1 and mk2 design is an increase in magnet size and the number of turns in the coil, all other dimensions remained the same. A summary of their key components and results are given in table 1 with comparisons to the current mk3 design. More details of the Mark 1 and 2 EM generator designs and results have been previously reported $[11,12]$.

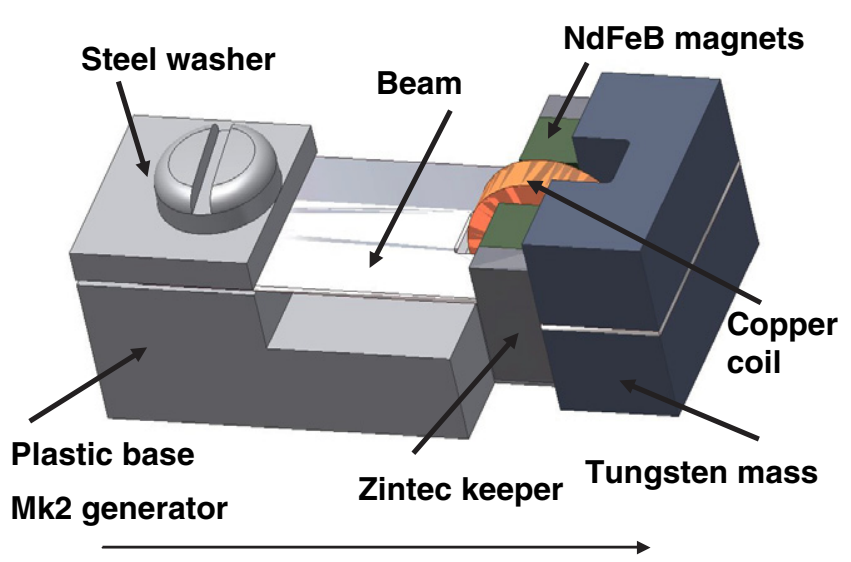

$10 \mathrm{~mm}$

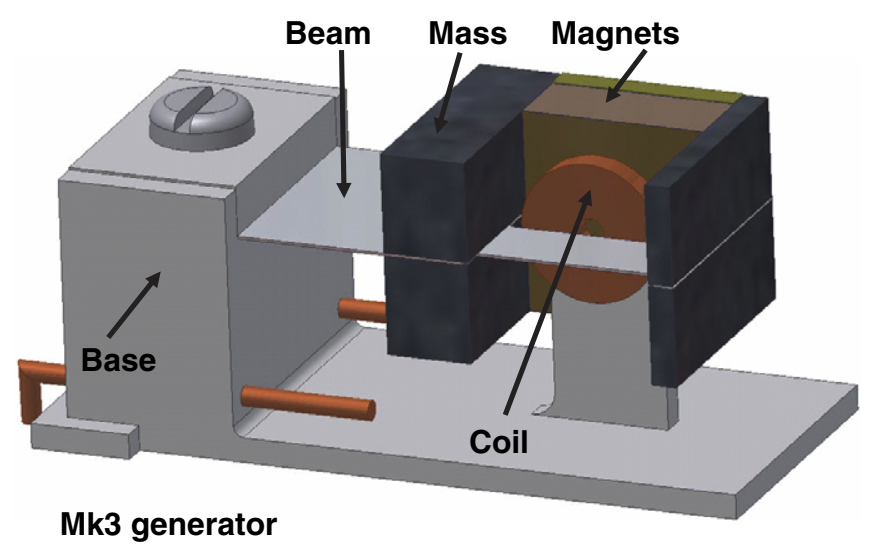

Figure 1. Previous $\mathrm{mk} 1 / 2$ generator and cutaway (two magnets and keeper removed) of the new $\mathrm{mk} 3$ generator design.

(This figure is in colour only in the electronic version)

The latest mk3 EM generator, shown in figure 1 with the mk1/2 design for comparison, is based upon the same cantilever beam arrangement. The NdFeB magnets, Zintec keepers and machinable tungsten masses have been bonded to the $50 \mu \mathrm{m}$ thick $\mathrm{BeCu}$ beam and positioned surrounding a stationary copper coil with 2800 turns wound from $12 \mu \mathrm{m}$ diameter wire.

The $\mathrm{mk} 3$ generator design has been improved in a number of ways compared to the previous generators. The cantilever structure was redesigned to enable manual tunability which would allow the generator to operate in different applications. Adjusting the resonant frequency of the generator is achieved by altering the cantilever beam length. ANSYS modal analysis predicted that an adjustment range of $1.3 \mathrm{~mm}$ would produce a frequency range of 44-60 Hz. Therefore, a slot of this length was etched into the beam at the clamping point to allow for manual adjustment of the beam length. In addition, the slot in 
the beam for the coil was increased in length to allow for this extra movement.

Increasing the velocity of the magnets, at a given frequency and acceleration level, and increasing the electromagnetic coupling would further improve the generated output voltage. To maximize the mechanical amplitude, and therefore the velocity, of the magnets in relation to the stationary coil whilst maintaining the resonant frequency, the inertial mass was moved closer to the clamping point and the magnets positioned as close to the beam tip as possible.

Ansoft Maxwell 3D software was used to model the coupled electromagnetic arrangement of the magnets and coil with the aim of increasing the electromagnetic coupling within the generator. This approach is necessary due to the complex geometry of the arrangement and to predict the coil voltage in conditions where the amplitude of the magnets might exceed the outer diameter of the coil. The length of the magnets was increased to $3.7 \mathrm{~mm}$ to ensure that the coil is always entirely within the magnetic flux irrespective of beam length adjustments. Finally, the horizontal gap between the magnets was reduced by $0.1 \mathrm{~mm}$, increasing the flux density by $12 \%$. This was the minimum gap size achievable whilst maintaining a reasonable yield of working generators.

Additionally, the rearrangement allowed for a marginally larger coil to be used whilst maintaining the same generator volume, $\sim 150 \mathrm{~mm}^{3}$. The new copper coil has outer and inner diameters of $2.8 \mathrm{~mm}$ and $0.5 \mathrm{~mm}$, respectively. It is wound with $12 \mu \mathrm{m}$ diameter copper wire achieving 2800 turns and a coil resistance of $2.3 \mathrm{k} \Omega$. Finally, the overall inertial mass was increased from $0.66 \mathrm{~g}$ to $1.02 \mathrm{~g}$ between the $\mathrm{mk} 2$ and 3 generators.

Because of the cyclical operation of the generator, the fatigue properties of the beam material have a particular significance. Beryllium copper was chosen for the beam material because it has high fatigue strength, $0.31 \mathrm{GN} \mathrm{m}^{-2}$ [13]. The maximum tip amplitude was identified using ANSYS static stress finite element analysis. A maximum beam tip amplitude of $1.8 \mathrm{~mm}$ (corresponding to a displacement of $0.85 \mathrm{~mm}$ at the centre of the magnets) ensures the fatigue limits of the material will not be exceeded. This result was used to calculate the remaining dimensions of the generator and was used in the Maxwell 3D magnetic model.

\section{System description}

The ACMS consists of three main modules: the EM micropower generator converting ambient vibration energy into electrical energy, the power management circuit which converts and stores the energy for later use and the microcontroller-based subsystem containing an accelerometer and RF transmitter. An overview of the ACMS is shown in figure 2 .

Typical applications for a system such as this would be in autonomous wireless condition monitoring, e.g., sensing bearing wear through vibration monitoring.

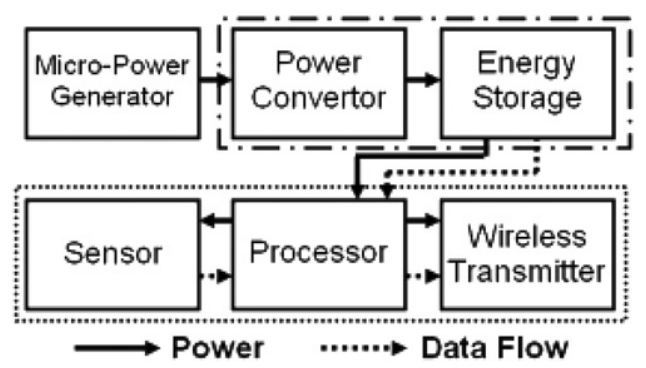

Figure 2. Block diagram for the autonomous wireless condition monitoring sensor system (ACMS).

\subsection{Power convertor and energy storage}

The generated voltage is insufficient to operate state of the art commercial off the shelf (COTS) low powered microcontrollers. Therefore, the voltage has to be increased to a suitable level greater than $2 \mathrm{~V}$ and the AC generator output must also be rectified.

A common solution is to use a charge pump circuit, also known as a voltage multiplier. There are two main configurations of voltage multiplier, the Villard cascade and the Dickson configuration [14]. The circuits operate by charging a capacitor in one half of the AC input cycle and then discharging it into the next stage of the circuit during the second half of the cycle. The number of stages in the circuit determines the multiplication level of the original input signal. Furthermore, the circuit behaves as a rectifier and therefore the final output is DC.

The energy produced by the voltage multiplier is stored within a supercapacitor. Supercapacitors offer higher capacity than conventional capacitors and do not suffer from the limited discharge cycles that batteries are subject to [15]. In addition, a supercapacitor avoids the extra circuitry and unnecessary power losses associated with a rechargeable battery. The microcontroller will draw energy from the supercapacitor to power the system when required and allow the supercapacitor to recharge between measurement/transmission cycles. The operation of the voltage multiplier is critical to the successful operation of the overall ACMS.

\subsection{Microcontroller subsystem}

The microcontroller subsystem consists of five modules: the PIC microcontroller, voltage reference, accelerometer, wireless transmitter and cold start circuit. It is essential that each of these modules exhibits low power consumption and a low operating voltage. Figure 3 shows the complete microcontroller subsystem.

The Microchip PIC16F676 was chosen as the low power microcontroller. The PIC16F676 is an 8 bit microcontroller with very low power consumption and it can operate at $2 \mathrm{~V}$. The standby current is low at just $1 \mathrm{nA}$ at $2 \mathrm{~V}$ and the operating current is $8.5 \mu \mathrm{A}$ at $32 \mathrm{kHz}, 2 \mathrm{~V}$. It has an on board watchdog timer with a current consumption of $300 \mathrm{nA}$ at $2 \mathrm{~V}$. Importantly, for measurement of the accelerometer data and supercapacitor voltage, it has an onboard 10 bit analogue-todigital (A/D) converter. This device is very flexible offering relatively straightforward field re-programmability. 


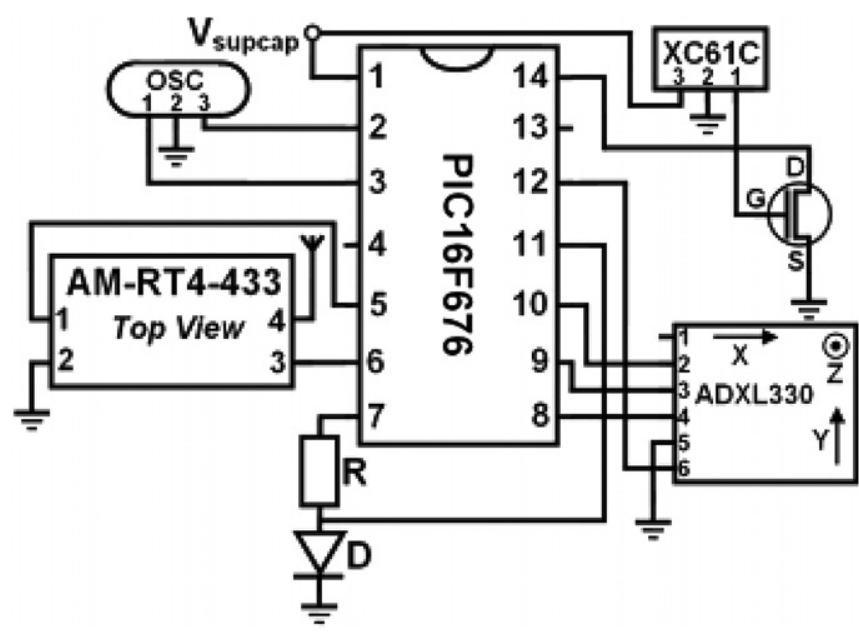

Figure 3. Microcontroller subsystem schematic.

In order for the ACMS to be energy aware, the system must monitor the energy available in the supercapacitor. The voltage on the supercapacitor is sampled and compared to a reference voltage $\left(V_{\text {ref }}\right)$ by the PIC. However, the default $V_{\text {ref }}$ on the PIC microcontroller is determined by the supply voltage (i.e., the voltage on the supercapacitor) and therefore it will effectively be comparing itself to itself and return a constant value. This was overcome by obtaining an alternative $V_{\text {ref }}$ which is based upon a diode/resistor potential divider. This provides a constant $V_{\text {ref }}$ due to the fixed voltage drop across the diode. The capacitor voltage is compared to this voltage reference and when the returned value matches or exceeds a predetermined level, the processor powers the rest of the system as required. The predetermined level is defined in the software and therefore this approach can be adjusted according to the particular energy requirements of different system configurations.

The accelerometer chosen was the Analog Devices ADXL330 because it had the lowest available operating voltage, $2 \mathrm{~V}$, whilst maintaining a high degree of sensitivity, $195 \mathrm{mV} / g$. The accelerometer requires an initialization and settling time of $20 \mathrm{~ms}$. Once settled, five accelerometer measurements are recorded by the PIC, and the peak acceleration value is transmitted. Radio-frequency communications are provided by the RF solutions AM-RT4 transmitter operating at $433 \mathrm{MHz}$. This offers a complete transmitter package with a very low operating current of $4 \mathrm{~mA}$ during transmission and can be supplied with data direct from the PIC.

Initial experiments showed that outside the specified voltage range the behaviour of the PIC changed. Problems occur because the PIC requires an increased initialization power and behaves unpredictably at voltages below $2 \mathrm{~V}$. This increased power usage would cause a drop in supercapacitor voltage so that the PIC turned off again; this cyclic situation meant that a stable operating stage was never reached. To overcome this problem, a Torex XC61C (power consumption $\sim 1.4 \mu \mathrm{W}$ ) voltage level detector, with a specified switching voltage of $2 \mathrm{~V}$, was used to control a CMOS switch which gated the power supply to the microcontroller. This extra circuitry, known as a cold start circuit, ensures that the PIC will only begin to draw power once a certain minimum supply voltage has been reached. In addition, the Torex XC61C has a degree of hysteresis in the switching value, so that it will not immediately turn off when the PIC draws energy and temporarily drops the supercapacitor voltage below $2 \mathrm{~V}$.

\section{Testing and results}

\subsection{EM generator}

The generator performance was evaluated using an accelerometer feedback controlled shaker unit, a programmable resistive load and a PC with LabView software collecting the data. This system allows the user to obtain reliable, repeatable results and program long sequences of tests to fully characterize the generator over a range of acceleration levels, load resistances and frequencies.

A key requirement for the generator design is a high degree of electromagnetic coupling between the movement of the magnets and the electrical signal from the coil; this is both to increase the amount of energy available electrically and to produce a voltage with an amplitude that is large enough to be rectified. The open-circuit coil voltage, $V$, is related to the velocity of the coil, $v$, by the equation

$$
V=K_{e} v,
$$

where $K_{e}$ is a measure of the electromagnetic coupling. For a straight sided coil in a uniform field of strength $B$, length of each coil turn in the field, $L$, and number of coil turns, $N, K_{e}=$ $B L N$. In our case, the magnetic field varies across the coil, the curvature of the coils is significant and $K_{e}$ is easiest to find by experiment — using a camera to measure beam amplitude at excitation levels measured by the accelerometer on the shaker unit. We measured it as $6.04 \mathrm{~V} \mathrm{~s} \mathrm{~m}^{-1}$.

The resonant frequency of the generator was obtained by sweeping through a range of frequencies. Once the resonant frequency was identified, the test was repeated with varying load resistances from $100 \Omega$ to $9 \mathrm{M} \Omega$. The generator was subjected to a vibration acceleration of $0.6 \mathrm{~m} \mathrm{~s}_{\mathrm{rms}}^{-2}$ throughout the tests. Figure 4 shows the maximum average power output of $58 \mu \mathrm{W}\left(931 \mathrm{mV}_{\mathrm{rms}}\right)$ which was achieved with a $15 \mathrm{k} \Omega$ load resistance with a resonant frequency of $50 \mathrm{~Hz}$. Figure 4 also includes comparative data for the $\mathrm{mk} 1$ and 2 generator power outputs into their respective optimum load resistances of $100 \Omega$ and $4 \mathrm{k} \Omega$. The Maxwell 3D modelling predicted a peak open-circuit voltage of $1.5 \mathrm{~V}$, whilst the practical generator produced $1.14 \mathrm{~V}$.

The mk3 generator output is summarized in table 1 . The generator demonstrated a $29 \%$ improvement in power output and $117 \%$ improvement in voltage output, when driving an optimal load resistance, compared to the mk2 generator $\left(428 \mathrm{mV}_{\mathrm{rms}}\right)$. The increased voltage is particularly important as the voltages from the $\mathrm{mk} 2$ generator could not be easily or efficiently rectified, and hence only a small fraction of the generated power was available in a useful form. The power output has not improved as significantly as the voltage because the increase in coil turns required to generate a higher voltage 


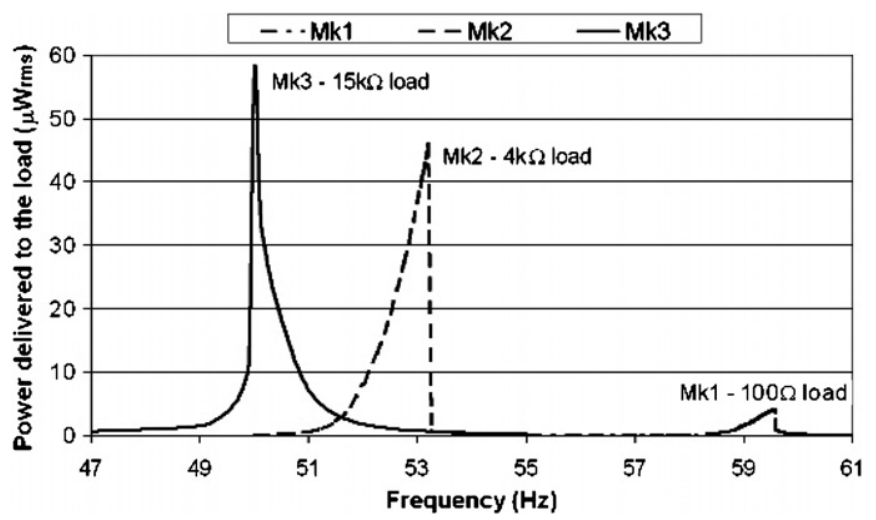

Figure 4. Generator output power driving an optimal load resistance for the $\mathrm{mk} 1,2$ and $3 \mathrm{EM}$ generators.

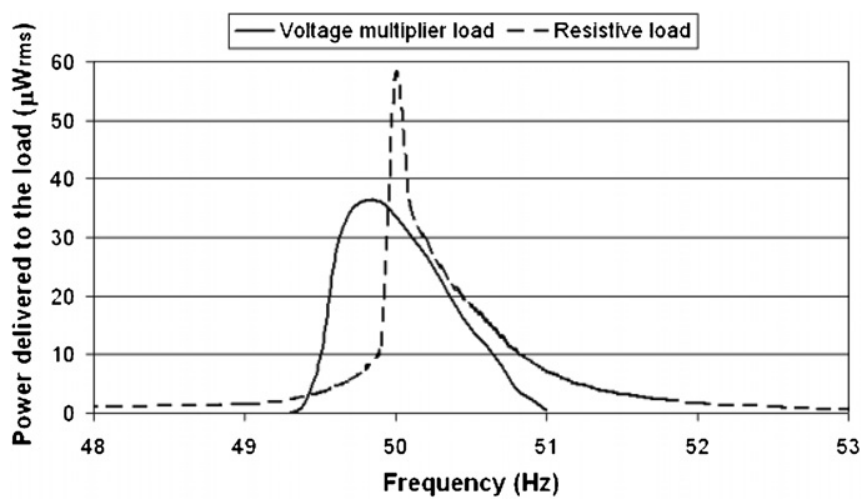

Figure 5. Maximum generated power by the $\mathrm{mk} 3$ generator into a resistive load of $15 \mathrm{k} \Omega$ and into the voltage multiplier circuit, both at $0.6 \mathrm{~m} \mathrm{~s}_{\mathrm{rms}}^{-2}$ vibration.

also increases the coil resistance, thus introducing additional power losses in the coil.

The generator was connected directly to the voltage multiplier circuit and the output power was measured using an opamp current monitoring circuit. Figure 5 shows the power delivered to the load when connected to the optimum resistive load and when connected to the voltage multiplier circuit with no load resistor. When connected to the voltage multiplier load at an acceleration level of $0.6 \mathrm{~m} \mathrm{~s}_{\mathrm{rms}}^{-2}$, the $\mathrm{mk} 3$ generator produces a peak output power of $36 \mu \mathrm{W}$.

The voltage multiplier improves the electromechanical conversion efficiency and thus increases the electrical damping on the generator. Therefore, when the generator is connected to the voltage multiplier, it can be driven to a higher acceleration level before it reaches the maximum beam amplitude compared to when driving a purely resistive load.

This effect was tested by increasing the acceleration level on the test rig to $1.7 \mathrm{~m} \mathrm{~s}_{\mathrm{rms}}^{-2}$ in order to achieve the same beam deflection (measured using a camera) as the resistive load test case. At this acceleration level, the average power output of the generator into the voltage multiplier is $120 \mu \mathrm{W}$, of which $77 \mu \mathrm{W}$ is delivered as useful energy to the supercapacitor. The low efficiency of the voltage multiplier circuit is a result of the reverse leakage currents of the diodes, combined with the leakage current of the capacitors.
Geometric nonlinearity is present within the generator due to the large deflection of the beam causing an increase in strain, thus a change in the beam length, as the beam achieves resonance [16]. This nonlinear effect saw the resonant frequency vary with excitation amplitude; around $0.5 \mathrm{~Hz}$ deviation in resonant frequency between 0.1 and $0.5 \mathrm{~m} \mathrm{~s}^{-2}$. Thus, simple mass spring-damper models presented previously [17] are not sufficient to provide accurate indications of the mechanical or electrical response of such generators. In particular, with devices working at resonance, where a small shift in the resonant frequency results in a large change in power output, the models can only provide a rough indication of the power that will be generated.

\subsection{Voltage multiplier (VM) and energy storage}

Electromagnetic vibration powered energy harvesting generators typically produce lower output voltages than their piezoelectric counterparts. In producing such a small generator, a particular challenge in this project was to successfully rectify and convert voltages of only a few hundred millivolts to useful voltage levels. To determine the optimum design for the VM, a number of circuits were compared using PSPICE circuit simulations and practical testing. Compared to a Villard cascade, the five-stage Dickson configuration was found to provide a larger output voltage and faster charging rate when connected to the electromagnetic generator. In addition, a range of common diode and capacitor values were evaluated. Schottky diodes were chosen for their characteristic properties of low turn on voltage and fast switching time. It is essential to maximize the power transferred from the generator to the supercapacitor, so switching losses must be kept to a minimum. It was therefore desirable to obtain a diode with a very low forward bias voltage. However, a reduced forward bias voltage is obtained at the expense of an increased reverse leakage current which in turn drives down the maximum voltage multiplier output. P-SPICE simulations identified the Siemens BAT760 diode as a good compromise solution. The BAT760 has a forward bias voltage, $V_{\mathrm{F}}$, of $\sim 100 \mathrm{mV}$ at $I_{\mathrm{F}}=0.1 \mathrm{~mA}$.

Analysis of the circuit revealed that $150 \mu \mathrm{F}$ capacitors at each stage increased the charging rate of the load supercapacitor. Compact surface mount $150 \mu \mathrm{F}$ tantalum oxide capacitors were identified as having the largest capacitance value whilst minimizing the PCB footprint.

Testing of the practical circuit revealed that the leakage current within the system was attenuating the generator output to such an extent that the advantage of larger capacitance and lower switching voltage was lost. Therefore, compact $100 \mu \mathrm{F}$ ceramic capacitors were used because these have a leakage current four times lower than the tantalum oxide capacitors. In addition, the improved voltage output from the $\mathrm{mk} 3$ generator allowed the use of Siemens BAT754 diodes, $V_{\mathrm{F}} \sim 200 \mathrm{mV}$ at $I_{\mathrm{F}}=0.1 \mathrm{~mA}$. Whilst these diodes have double the turn on voltage they reduce the leakage current by a factor of 10 .

Panasonic $0.22 \mathrm{~F}, 0.1 \mathrm{~F}$ and $0.047 \mathrm{~F}$ supercapacitors were tested with the voltage multiplier. The $0.047 \mathrm{~F}$ supercapacitor was identified as the optimum solution as it charged up quickly 


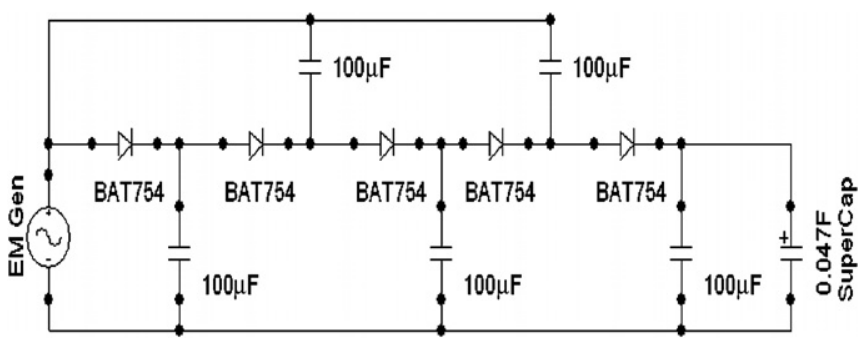

Figure 6. Final optimized voltage multiplier circuit.

yet still stored sufficient energy to maintain a stable energy supply for the PIC during the measurement cycle. The time required to charge it from 0 to $2.2 \mathrm{~V}$ was $4 \mathrm{~h}$ compared to $7 \mathrm{~h}$ for the $0.22 \mathrm{~F}$ supercapacitor.

These improvements, combined with the improvements in the mk3 generator, produced a voltage multiplier efficiency of $65 \%$ and an output voltage in excess of the $2.2 \mathrm{~V}$ required by the rest of the system. This corresponds to a maximum available power into the supercapacitor of $23.4 \mu \mathrm{W}$ with $0.6 \mathrm{~m} \mathrm{~s}^{-2}$ acceleration. The final circuit is shown in figure 6 .

\subsection{Microcontroller subsystem}

The PIC is programmed to operate in a low power sleep mode until the supercapacitor voltage reaches $2.2 \mathrm{~V}$. The PIC periodically samples the supercapacitor voltage and once this threshold is reached, indicating that sufficient power is available, the PIC switches on the accelerometer. The PIC then takes 16 measurements at a sampling rate of $1.25 \mathrm{kHz}$ from the accelerometer and records the peak acceleration value. The transmitter module is then switched on and transmits a 17 bit packet consisting of a synchronization bit, eight identification bits and the 8 bit acceleration data value.

Initially the PIC was programmed to switch on the accelerometer for five individual measurements, switching off the accelerometer between measurements. However, power consumption results showed that the accelerometer required a significant amount of power to initialize. Therefore, the accelerometer actually used less power when it was switched on just once but taking 16 measurements during that time period. This change in the code reduced the overall system power consumption from $109 \mu \mathrm{W}$ to $21 \mu \mathrm{W}$ and produced more consistent measurements. The full timing and power consumption measurements for the microcontroller subsystem are shown in table 2. Table 2 shows that the accelerometer consumes $80 \%$ of the total energy per cycle with the rest of the circuit and in particular the data transmission having less impact on the total energy consumption.

The cold start circuit was tested by charging the supercapacitor from zero with and without the cold start circuit, and the results of this test are shown in figure 7 . Without the cold start circuit the supercapacitor could only be charged to $0.9 \mathrm{~V}$, and the system never became fully functional (as described in section 3.2).

Finally, the entire sensor system was tested and then demonstrated on both an air compressor and an office air

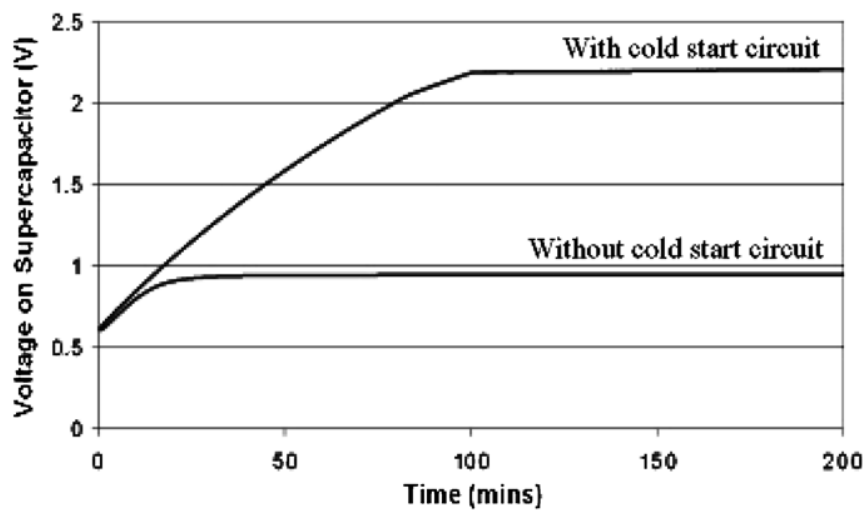

Figure 7. Supercapacitor voltage with and without cold start circuit.

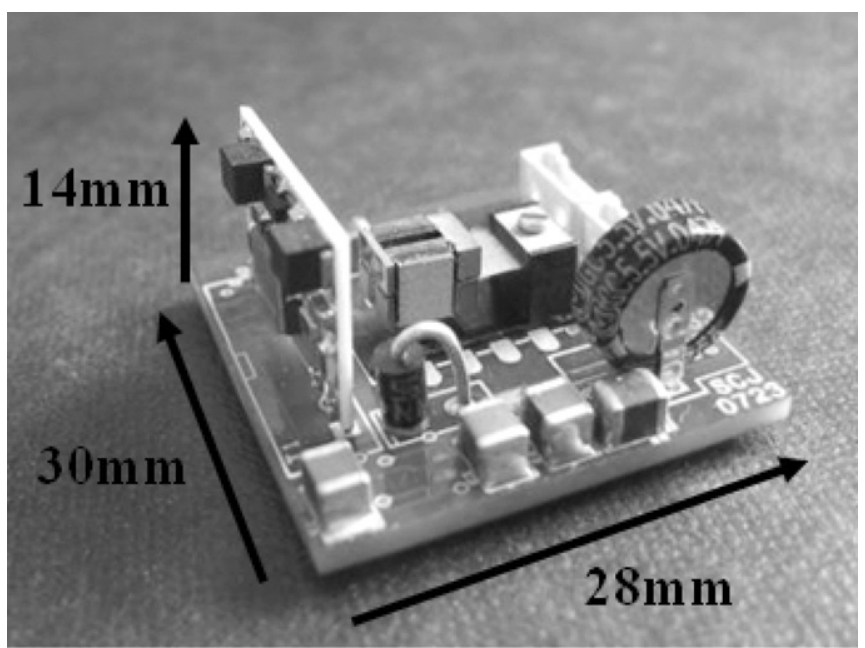

Figure 8. Wireless sensor system with $\mathrm{mk} 3$ generator attached.

Table 2. Power consumption for each programme state.

\begin{tabular}{llll}
\hline State & Time & $\begin{array}{l}\text { Average power } \\
\text { during operation }\end{array}$ & Energy/cycle \\
\hline Reference voltage & $68 \mu \mathrm{s}$ & $1.95 \mathrm{~mW}$ & $133 \mathrm{~nJ}$ \\
Sensor reading & $32.7 \mathrm{~ms}$ & $1.62 \mathrm{~mW}$ & $51.9 \mu \mathrm{J}$ \\
Transmit & $9 \mathrm{~ms}$ & $2.4 \mathrm{~mW}$ & $10.8 \mu \mathrm{J}$ \\
Sleep mode & $3.24 \mathrm{~s}$ & $0.784 \mu \mathrm{W}$ & $2.54 \mu \mathrm{J}$ \\
Total duty cycle & $3.28 \mathrm{~s}$ & $21.3 \mu \mathrm{W}$ & $65 \mu \mathrm{J}$ \\
\hline
\end{tabular}

conditioner unit transmitting a peak acceleration value every $3 \mathrm{~s}$ in both cases. Both applications had resonant peaks between 40 and $50 \mathrm{~Hz}$ with acceleration levels between 0.85 and $1.7 \mathrm{~m} \mathrm{~s}^{-2}$. The ACMS was operated continuously for $10 \mathrm{~h}$ and transmitted data throughout this time. Sufficient power is available for the transmitter to transmit data to a receiver module at a range of $20 \mathrm{~m}$. Figure 8 shows the completed wireless sensor system with an $\mathrm{mk} 3$ generator attached (the circuit board measures $28 \times 30 \mathrm{~mm}^{2}$ ). The PCB is currently attached to the application using a magnetic mount.

\section{Discussion of results}

The results show that the voltage multiplier circuit is able to extract more power from the generator, compared to a 
simple load resistor, by significantly increasing the electrical damping (the $Q$-factor of the electrically damped beam is decreased from 210 to 60), thus allowing a larger input vibration amplitude. The damping is further increased by the improved electromagnetic coupling for the $\mathrm{mk} 3$ generator $\left(K_{e}=6.04 \mathrm{~V} \mathrm{~s} \mathrm{~m}^{-1}\right)$ compared to the mk2 design $\left(K_{e}=\right.$ $\left.4.43 \mathrm{~V} \mathrm{~s} \mathrm{~m}^{-1}\right)$ at the same applied acceleration level of $0.6 \mathrm{~m} \mathrm{~s}^{-2}$. This is clearly shown in figure 5 by the broader, lower amplitude resonant peak. A common problem with vibration powered generators is that in order to extract maximum power they are designed to work at resonance; this resonance is often associated with a high $Q$-factor, and hence a narrow band of frequencies over which the device can generate useful power. As environmental conditions vary, especially temperature, the resonant frequency of the generator can drift causing it to no longer match the frequency of the application vibrations. Therefore, the broader bandwidth response allows the generator to work over a wider range of vibration frequencies and is preferable in many practical applications.

The generator structure limits the beam motion using over range protection provided by the base and lid. The generator has been designed to be limited to tip beam deflections of $1.8 \mathrm{~mm}$. With a resistive load this is reached at an acceleration level of $0.6 \mathrm{~m} \mathrm{~s}^{-2}$. However, when connected to the voltage multiplier circuit the generator can be driven up to an acceleration level of $1.7 \mathrm{~m} \mathrm{~s}^{-2}$ before reaching maximum deflection at which point the power output is $120 \mu \mathrm{W}$.

The $\mathrm{mk} 3$ electromagnetic generator achieves a conversion efficiency of $\sim 53 \%$ when connected to the voltage multiplier circuit [18] which is much greater than that achievable with alternative piezoelectric generators. The maximum efficiency of piezoelectric vibration energy harvesters is limited by the electromechanical coupling coefficient. For bulk piezoelectric ceramics the maximum efficiency is $35 \%$ for PZN-PT when used in the $\mathrm{d} 33$ mode. This reduces to $7 \%$ when used in the d31 mode [19]. The electromagnetic transduction principle is not limited in this way and much higher efficiencies can be achieved.

One simple figure of merit that can be used to analyse generator performance is normalized power density (NPD). Comparative values of NPD for a variety of energy harvesting transduction methods have been previously reported by Beeby et al [12]. The NPD figure is obtained calculating the power output of the generator normalized to acceleration level and dividing by the device volume. It is not a perfect tool for comparison since it does not take into consideration the bandwidth of the device. This means the NPD figure benefits high $Q$, narrow bandwidth devices which are of limited use in practice. It does, however, provide a simple figure of merit that can be calculated from the type of data typically available in the literature. The $\mathrm{mk} 3$ generator has an NPD of $1115 \mathrm{~kg} \mathrm{~s} \mathrm{~m}^{-3}$ with a resistive load and $288 \mathrm{~kg} \mathrm{~s} \mathrm{~m}^{-3}$ peak with the voltage multiplier load (due to the increased electrical damping and bandwidth, and the corresponding reduction in peak power). This compares very favourably with an NPD of $60 \mathrm{~kg} \mathrm{~s} \mathrm{~m}^{-3}$ for a similar piezoelectric generator by Roundy et al [4].

The generator was able to charge a supercapacitor in $4 \mathrm{~h}$ from a cold start with a continuously applied acceleration level of $0.6 \mathrm{~m} \mathrm{~s}^{-2}$. The system is energy aware and will change the measurement/data transmission duty cycle, therefore altering its power consumption, depending on the amount of energy being harvested. This represents the ability of the system to adapt to fluctuations in the amount of vibration energy available from the intended application. The system still works at acceleration levels as low as $0.2 \mathrm{~m} \mathrm{~s}^{-2}$ at which point it transmits data once every $12 \mathrm{~min}$.

These results compare very favourably with a similar wireless sensor system developed by Yuen et al [20]. Their AA battery sized device uses an EM generator to convert a vibration acceleration of $4.63 \mathrm{~m} \mathrm{~s}^{-2}$ at $70.5 \mathrm{~Hz}$ to $60 \mu \mathrm{W}$ of power. This is almost a factor of 8 increase in vibration acceleration level to achieve a similar amount of power as the ACMS. Their system monitors temperature and can transmit data every $18 \mathrm{~s}$ with an operating power consumption of $27.6 \mu \mathrm{W}$.

\section{Conclusions and further work}

This paper has successfully demonstrated an autonomous wireless condition monitoring sensor system powered by harvested vibrational energy. The electromagnetic generator has a volume of only $150 \mathrm{~mm}^{3}$ but produces sufficient electrical energy, from a very low vibration source of $0.2 \mathrm{~m} \mathrm{~s}^{-2}$, to power the entire microcontroller subsystem with no additional battery or power supply. The ACMS is energy aware and will therefore alter its power usage depending on the amount of energy available. The results presented here have particular significance because the low level of vibration required to power the system can be widely found in many environments. In particular, the frequency and vibration levels available here are widespread in industrial and domestic machinery.

The ACMS is currently able to measure acceleration data and transmit the result typically every $3 \mathrm{~s}$ when excited by vibration levels of $0.6 \mathrm{~m} \mathrm{~s}^{-2}$. The minimum duty cycle is limited to $3 \mathrm{~s}$ by the PIC programme to allow sufficient excess charging of the supercapacitor. Overcharging the supercapacitor in this manner provides a reservoir of stored energy which acts as a buffer to ensure a reasonably stable period of operation even when the generator is not operating at its maximum output level. This minimum duty cycle can be reduced by adjusting the watchdog timer where required or alternatively the amount of measured data within this period could be increased to provide more information for the receiver. Since there is surplus energy, future work will consider the integration of data processing into the ACCM and alternatively the transmission of larger amounts of data so that additional processing (e.g. a fast Fourier transform) can be performed at the receiver, thus improving the functionality of the system.

\section{Acknowledgments}

The authors would like to acknowledge the funding from the EU (grant number FP6-IST-507911) for the project VIBES under which this work was carried out. 


\section{References}

[1] Banazwski B and Shah R K 2003 The role of fuel cells for consumer electronic products and toys Proc. 1st Int. Conf. on Fuel Cell Science, Engineering and Technology (Rochester, NY) pp 149-55

[2] Epstein A H 2004 Millimetre-scale, micro-electro-mechanical systems gas turbine engines J. Eng. Gas Turbines Power $126205-6$

[3] Beeby S, Tudor M and White N 2006 Energy harvesting vibration sources for microsystem applications Meas. Sci. Technol. 17 R175-95

[4] Roundy S and Wright P 2004 A piezoelectric vibration based generator for wireless electronics Smart Mater. Struct $131131-42$

[5] Arnold D P 2007 Review of microscale magnetic power generation IEEE Trans. Magn. 43 3940-51

[6] Roundy S, Wright P K and Rabaey J 2003 A study of low level vibrations as a power source for wireless sensor nodes Comput. Commun. 26 1131-44

[7] Torah R N, Tudor M J, Patel K, Garcia I N and Beeby S P 2007 Autonomous low power microsystem powered by vibration energy harvesting IEEE Sensors'07 Atlanta, Digest of Technical Papers pp 141-2

[8] http://www.perpetuum.co.uk, webpage accessed on 12 May 2008

[9] Glynne-Jones P, Tudor M J, Beeby S P and White N M 2004 An electromagnetic, vibration-powered generator for intelligent sensor systems Sensors Actuators A $110333-49$

[10] Beeby S P, Tudor M J, Torah R N, Koukharenko E, Roberts S, O'Donnell T and Roy S 2006 Macro and micro scale electromagnetic kinetic energy harvesting generators Proc. DTIP Conf. (Stresa, Italy)

[11] Torah R N, Beeby S P, Tudor M J, O'Donnell T and Roy S 2006 Development of a cantilever beam generator employing vibration energy harvesting PowerMEMS'06, Digest of Technical Papers pp 181-4

[12] Beeby S P, Torah R N, Tudor M J, Glynne-Jones P, O'Donnell T, Saha C R and Roy S 2007 A micro electromagnetic generator for vibration energy harvesting J. Micromech. Microeng. 17 1257-65

[13] NGK Berylco UK Ltd, http://www.ngkberylco.co.uk/ charact.htm, webpage accessed on 24 September 2008

[14] Pan F and Samaddar T 2006 Charge Pump Circuit Design (New York: McGraw-Hill)

[15] Jiang X, Polastre J and Culler D 2005 Perpetual environmentally powered sensor networks Proc. 4th Int. Symp. on Information Processing in Sensor Networks

[16] Arafat H N 1999 Nonlinear response of cantilever beams PhD Thesis Virginia Polytechnic Institute and State University, Blacksburg, Virginia

[17] Williams C B and Yates R B 1996 Analysis of a micro-electric generator for microsystems Sensors Actuators A 52 8-11

[18] Torah R N, Glynne-Jones P, Tudor M J and Beeby S P 2007 Energy aware wireless microsystem powered by vibration energy harvesting Proc. PowerMEMS 2007 Conference (Freiburg, Germany)

[19] Roundy S 2005 On the effectiveness of Vibration-based Energy Harvesting J. Intell. Mater. Syst. Struct. 16 809-23

[20] Yuen S C L, Lee J M H, Li W J and Leong P H W 2007 An AA-sized vibration-based microgenerator for wireless sensors IEEE Pervasive Comput. 6 64-72 